\title{
Use of modelling to inform public health policy: a case study on the blood-borne transmission of variant-CJD
}

\begin{abstract}
Since the identification of variant Creutzfeldt-Jacob Disease (vCJD) in the late 1980s, the possibility that this disease might be passed on via blood transfusion has presented challenging policy questions for Government and blood services in the UK. This paper discusses the use of mathematical modelling to inform policy in this area of health protection. We focus on the use of a relatively simple analytical model to explore how many such infections might eventually be expected to result in clinical cases under a range of alternative scenarios of interest to policy, and on the potential impact of possible additional counter measures. We comment on the value of triangulating between findings generated using distinct modelling approaches and observational data.
\end{abstract}

Key words: Public health; Government; Risk.

\section{Introduction}

Variant Creutzfeldt-Jacob Disease (vCJD) is a fatal neurological disorder associated with the presence of an abnormal, 'misfolded' form of prion protein (Collinge 2001). It almost certainly first spread to humans via cattle infected with Bovine Spongiform Encephalopathy (BSE), otherwise known as "Mad Cow" disease (Bruce et al. 1997; Hill et al. 1997). At the time of writing, there have been 176 definite or probable vCJD cases in the UK and cases have occurred in several other countries, France having reported over twenty (UK NCJDRSU \& UK Blood Services 2011). Those affected have typically been young, with the UK cases having a median age of about 28 years at onset of symptoms.

Earlier fears of large numbers of vCJD deaths, based in part on mathematical epidemiology (e.g. (Ghani et al. 1998; Ghani et al. 2000)), have not been realised and incidence of new cases has been in decline following a peak of 29 in 1999. However, concern remains that many more people might have been infected, while not showing any 
symptoms. The potential risk of "secondary" (person-to-person) transmission could therefore be greater than implied by the small number of cases seen so far.

\section{Secondary infection of $v C J D$ via blood transfusion}

This analysis concentrates on transmission of vCJD via red cell units, though it should be noted that these do not consist of "pure" red cells, but also contain some plasma and white cells. Each unit of red cells transfused exposes the recipient to a substantial volume of material from a single donor.

Until 2003, there was no direct evidence as to whether blood from a donor incubating vCJD could infect the recipient, though similar prion diseases had been transmitted via blood transfusion in various animal models (e.g. (Gregori et al. 2004; Houston et al. 2008)). Unfortunately, the reality of vCJD transmission via blood is now established. Three clinical cases diagnosed between 2003 and 2006 had previously received red cell transfusions from donors later diagnosed with vCJD (Llewelyn et al. 2004; Wroe et al. 2006; Hewitt et al. 2006; Head et al. 2009). Two of these had received blood from the same donor. These three cases are therefore presumed to have been caused by bloodborne infection. Evidence of sub-clinical prion infection was found at autopsy in another recipient linked to an infected donor (Peden et al. 2004).

From the first identification of vCJD, UK policy had been based on the presumption that infection might be transmissible from person to person and various precautionary steps have been taken to protect the blood supply (Bennett \& Dobra 2006). Most relevant here, is that the processing of donations into red cell units has involved removal of white cells (leucodepletion) since 1999 to reduce any vCJD infectivity present in these cells. Although this is currently considered unlikely to eliminate the risk entirely (Gregori et al. 2004; Houston et al. 2008), all the transmissions identified so far took place prior to this step. From 2004, recipients of blood components have been excluded from donating blood in order to prevent vCJD, or any other infection, being "recycled" within the population. 
vCJD epidemiology: predictions and observations

Despite a great deal of research on both the science and the epidemiology of vCJD, great uncertainties remain. In particular, it is unclear how many of those infected are likely ever to develop clinical disease, and if so in what timescale. It appears that genotype plays a key role in this. To date, every "definite" or "probable" vCJD case in which genotype has been established has been "methionine-methionine homozygous" (MM) at Codon 129, a genotype present in $40 \%$ of the UK population. However, evidence of subclinical infection has been found in the other genotypes (Peden et al. 2004; Ironside 2006; Peden et al. 2010), as has one unconfirmed clinical case classed as "possible" vCJD (Kaski et al. 2009). In other acquired prion diseases (e.g. Kuru in humans and scrapie in sheep), cases have occurred in all genotypes but typically with different incubation periods, which can sometimes span decades (d'Aignaux et al. 2002; Collinge et al. 2006).

Concern thus remains that further waves of clinical cases will be seen in other genotypes, whilst the extent of any "silent" vCJD infection remains unclear (Clarke \& Ghani 2005; Bishop et al. 2006). There may be a significant population of infected individuals who die having never developed clinical vCJD. This "carrier state" would help explain the mismatch between the small number of clinical cases observed so far and the scale of exposure to BSE in the food chain. Nevertheless, these individuals might act as sources of onward infection, eventually leading to further secondary cases.

\section{Policy questions and the role for OR modelling}

Given the scientific uncertainties and degree of government responsibility, policy decisions have needed a strongly precautionary element. Nevertheless, as far as possible they also need to be proportionate to the potential risk, effective (and appropriately costeffective) in plausible scenarios, and appropriately prioritised. Reconciling these criteria is very challenging (Watkins et al. 2011). The small number of clinical cases seen so far raises legitimate questions as to how great a public health risk vCJD now poses (see e.g. (Lefrère \& Hewitt 2009; Will 2010; Wallis 2011; Dodd 2010; Knight 2010)). Modelling 
has helped inform policy by setting out the potential scale of secondary infection in alternative scenarios and the effectiveness of risk reduction steps, using ranges of inputs drawn from a mixture of basic scientific research, modelling and expert judgement. For example, the decision to exclude blood recipients from donating was influenced by system dynamics modelling of "feedback" of infection within the population (Bennett \& Dobra 2006). OR is being used to assess the implications of a screening blood test for vCJD, should efforts to develop one succeed (Department of Health 2009), and has also informed measures to reduce the risk of vCJD being transmitted through re-use of surgical instruments (Bennett et al. 2004, Stevenson et al. 2009).

Whilst policy has generally been informed by models that use "precautionary" inputs, the passage of time has made it more meaningful to "calibrate" transmission models against observed numbers of cases. Given the small number of clinical vCJD cases so far that might credibly have been due to blood-borne infection, how many future cases might plausibly occur and how would this be affected by further counter measures?

This paper reports on OR modelling conducted in response to an unfolding debate among decision makers about these issues. The work provided a means of analytically exploring the underpinning research question: for blood-borne transmission of vCJD, what combinations of plausible scientific assumptions on model inputs can be made consistent with the observed numbers of clinical cases?

\section{Methods}

Using OR to inform policy: a collaborative approach

The UCL Clinical Operational Research Unit (CORU) and the Department of Health's (DH) Health Protection Analytical team (HPAT) have a history of collaboration in developing analysis for health protection policy. This has typically involved an initial phase of discussion about the nature of the problem and the decision-making processes that could be informed by the research. Frequent liaison between the two teams and DH policy colleagues informs the choice of approach and any simplifying assumptions required. However, in some cases CORU staff have undertaken initial model 
specification without first seeing details of work already done by the DH team. This informal "blinding" allows a degree of independence, so that the approaches taken can be compared. Model development itself is typically iterative and uses bespoke tools developed by CORU. This project followed a similar pattern.

\section{Rationale for developing a simple model}

Existing HPAT models of blood-borne vCJD transmission were based on separate inputs that were each consistent with the available evidence and expert scientific advice. However, precautionary assumptions taken in combination can lead to scenarios that markedly over-estimate the numbers of cases due to transfusion that would have been seen so far. This problem of "model calibration" had long been recognized, but it was much less clear which (combinations of) parameters might need to be changed. With the accumulating epidemiological, clinical and experimental observations, the need to identify a feasible and consistent range of scenarios had become increasingly pressing.

Independently, other researchers had used state-of-the-art epidemiological modelling of the entire vCJD outbreak for a similar purpose. This involved sampling of multiple model parameters within a simulation framework, constrained to produce output consistent with observed prevalence of infection and numbers of cases (Garske \& Ghani 2010). Overall results are based on running the simulation over one million times and generating posterior likelihood estimates for the resulting scenarios. Although powerful, this approach does not provide an easy way of exploring the effects of different assumptions or parameter estimates concerning individual steps in the causal chain from primary infection through secondary infection via transfusion to subsequent clinical cases.

It was considered that there would also be value in relatively simple modelling designed to create a transparent framework for working through the consequences of alternative beliefs around key mechanisms and parameters. In the context of informing policy, this "story board" approach could then be used as a third point to triangulate the results of the more complex simulation model and actual observations, and to communicate the key 
messages. Such a model could also be modified rapidly if new information came to light that modified existing assumptions, as indeed happened in the course of this work.

\section{Project life cycle}

The work proceeded in three phases. Firstly, an initial mathematical model for estimating the number of secondary infections and clinical cases of vCJD by calendar time was developed (Figure 1). Secondly, the model was used to explore illustrative scenarios that were presented to a Government advisory committee. Finally, the feedback from this advisory committee was addressed as part of a process of revising the Government's assessment of secondary vCJD transmission via blood transfusion.

\section{Phase 1: Developing the mathematical model}

The first task was to develop a model to estimate the number of secondary infections over calendar time, taking account of possible variations in primary vCJD prevalence by birth cohort (Figure 1). The model therefore split the general population into birth cohorts (and by gender), allowing primary vCJD prevalence to vary between these. The timedependence of primary infection was modelled using a Normal distribution, the mean and variance of which are inputs chosen by the user. The mean interval between primary infection and onset of infectivity in blood for the general population could also be varied: this is important because delayed onset of infectivity will remove earlier secondary infections and cases. Other input determines the provenance of donated blood units in terms of the age and sex profile of the donor population.

Transfusion recipients are classified as "acute" or "chronic", the former typically undergoing one-off transfusion (e.g. following an accident, or during surgery), the latter undergoing repeated transfusions necessitated by ongoing medical conditions. These are considered separately due to the different patterns of transfusion and survival: for example many "acute" recipients will have life-expectancy typical of their age, but only if they survive the immediate episode necessitating the transfusion. 
Although secondary infections are important, what is of most concern, and can be compared with observation, is the number and timing of clinical (i.e. symptomatic) vCJD cases. To estimate the number of clinical cases appearing by calendar year as a result of blood transfusion, the key additional inputs concern the survival of acute and chronic recipients and the incubation periods between infection and onset of clinical vCJD. The former can be informed by empirical data, though long-term survival after transfusion is subject to some uncertainties. However, very little is known about the possible range of incubation periods. Although the three presumed transfusion-associated cases all developed symptoms well within 10 years of transfusion, we do not know how typical this is. Therefore, within the model, the user is able to choose both the mean and (gamma) distribution for secondary incubation periods, taking MM homozygotes and other genotypes separately, and to vary the proportion of infected recipients susceptible to developing clinical vCJD at all.

Taken in sequence, the discrete modules or steps within the overall model provide a way of working through the consequences of alternative inputs about the uncertain mechanisms and parameters. The model is relatively simple and does not attempt to capture every possible aspect of vCJD transmission risk. Nevertheless, it provides a way of rapidly exploring what happens at each step as individual inputs and assumptions are varied. The model illustrated here deals only with transmission via red cell units, although much of the same logic applies to transfusion of other components.

In presenting estimated numbers of clinical cases in different scenarios, it is important to distinguish between clinical cases that would have already occurred (providing the comparison with observations to date) and those projected to occur in the future. Amongst the latter, we need to distinguish between cases arising from infections that would already have happened and those caused by infections that could potentially be avoided through future policy decisions.

\section{Phase 2: Initial exploration of scenarios}


Before exploring the potential impact of policy options, an essential first step is to establish a plausible range of "baseline" scenarios for the number of clinical blood-borne secondary vCJD cases in the absence of further interventions. Given all the uncertainties inherent in this problem, the aim was to populate the model with inputs that were consistent with current scientific evidence and which also resulted in model outputs broadly consistent with the number of observed clinical vCJD cases plausibly attributable to transfusion. These questions of consistency are not straightforward, as discussed in greater depth elsewhere (Department of Health 2011).

Some inputs could be based on empirical data. For example, we adopted a static distribution for the provenance of donated blood units by age of donor, based on UK blood services studies used in previous HPAT analysis (Department of Health 2006). Inputs on post-transfusion survival could be informed by existing studies (Wallis et al. 2004; Llewelyn et al. 2004; Wells et al. 2009): extrapolating these suggests that about $25 \%$ of red cell units go to recipients surviving at least 10 years. Other initial assumptions were based on existing advice from scientific committees convened by DH. Notably, the scientific consensus, albeit based largely on animal models (Houston et al. 2008), was that a unit of infected red cells would contain more than enough infectivity to transmit vCJD. Given this advice, we initially regarded transmission as certain.

Of the uncertainty affecting other inputs, those around secondary incubation periods were significant. In initial illustrative scenarios we used mean incubation periods of 10 years for susceptible MM homozygous genotypes and 20 years for others. Whilst these values were compatible with the observed evidence and with literature on other prion diseases, we explored the sensitivity of model results to the choice of shape and scale parameters of the gamma distributions used.

Using these provisional inputs, we then explored scenarios based on differing levels of prevalence of primary vCJD infection by birth cohort. The evidence most widely used to estimate prevalence was a retrospective study in which appendix samples were tested for abnormal prion protein (Hilton et al. 2004). This found 3 positive samples in approximately 12,000, suggesting a prevalence of abnormal prion protein of about 1 in 
4,000 , with a $95 \%$ confidence interval of roughly 1 in 1,200 to 1 in 20,000 . The precautionary assumption is that presence of abnormal prion protein in appendix (or other lymphoid tissue) would be indicative of infectivity in blood. The large majority of the appendices in this study were from patients born between 1960 and 1985, and there was considerable uncertainty as to whether the results should be taken to apply only to this birth cohort, or to all cohorts born before 1996 (by which point precautions to protect the human food chain should have largely eliminated the risk of primary infection from BSE). With this in mind, we explored three different scenarios for the prevalence of infective donors:

(a) 1 in 4,000 for all age cohorts born up to 1996 ;

(b) 1 in 20,000 for all such cohorts;

(c) 1 in 20,000 in the $1960-1985$ birth cohort and close to zero in all others.

As outlined below, more recent evidence on prevalence suggest that (b) and (c) can no longer be regarded as realistic. Nevertheless, the results derived from all three remain of some interest. As an initial illustration, we chose model inputs to reflect primary infections occurring circa 1990 and allowed for a mean delay of 2 years in blood becoming infective. With a prevalence of infective donors of 1 in 4,000, the model shows a very large number of transmissions (over 500 per year) taking place from the mid-1990s onward. The rate only tails off as more donors come from amongst those born from 1996 onward.

To model the appearance of clinical cases, we used the inputs set out earlier for incubation periods (using Gamma distributions with shape parameter $=10$ and 20 years for MMs and other genotypes respectively, and scale parameters= 1 in this initial illustration). Projected case numbers would then be as shown in Figure 2 if all recipients are susceptible to clinical disease, the lower curve indicating those developing clinical vCJD before dying of some other cause. The resulting scenario is clearly unrealistic, predicting over 50 blood-borne clinical vCJD cases occurring annually since about 2005, and approximately 600 to date. This example illustrates the severity of the "model calibration" problem. Inputs that reflected the best scientific knowledge and advice on 
each separate factor could produce outputs that seriously exaggerate the number of clinical cases seen to date.

Prevalence scenario (b), applying a blanket estimate of 1 in 20,000, produces estimates lower by a factor of 5. Scenario (c), applying this prevalence to the 1960-85 birth cohort only, represented the "minimal" consistency with the Hilton et al. result required if presence of abnormal prion in tissue is equated with infectivity in blood. The number of secondary infections is reduced by a further factor of about 2 . In addition, a higher proportion of transmissions would occur later, with the resulting incidence of clinical cases peaking between 2020 and 2025, as shown in Figure 3.

Even in this scenario, a significant problem of over-prediction remained. However, the main interest in scenarios such as this lies in comparison with the Garske and Ghani model, which constrained prevalence of infection in a similar way. Scenario (c) (Figure 3) reproduces some of the key features of that model's central scenario, producing a long wave of secondary cases with relatively small numbers appearing each year but persisting for several decades. Consistency both with the Garske and Ghani model and with the case data could be improved by allowing susceptibility to clinical vCJD to be less than $100 \%$. Confining susceptibility to about $40 \%$ of recipients produces scenarios similar to the "central" Garske and Ghani scenarios of between 250 and 300 blood-borne cases in total, depending on the assumed effect of leucodepletion. The similarity could be increased further through, for example, increasing the mean delay in blood becoming infective. This consistency in results using different modelling methods provided some reassurance as to the robustness of the approach.

\section{Phase 3: Discussions with Government advisory committee}

The continuing need to establish a credible range of scenarios for blood-borne transmission of $\mathrm{vCJD}$ prompted a comprehensive re-examination of the existing evidence and projections At the request of DH, this was led by the Transmissible Spongiform Encephalopathy (TSE) Risk Assessment Subgroup of the Advisory Committee on Dangerous Pathogens (ACDP). This specialist scientific committee (referred to as "the 
Subgroup" in what follows) includes leading experts in prion diseases, representatives of the UK blood services, independent epidemiologists and modellers. The Subgroup's first discussion was informed by the DH paper already referred to (Department of Health 2011). This set out the calibration problem, summarised the evidence available both on model inputs and on case data against which outputs could be compared, and presented illustrative model results. As summarised in the minutes of the meeting (ACDP 2011), the ensuing discussion led to the following key conclusions:

- The Subgroup agreed that transmission models should now be calibrated against observed case numbers. More specifically, "realistic" scenarios for transmission via blood components should result in between 3 and $10 \mathrm{vCJD}$ cases with onset of symptoms occurring prior to 2011. (The precautionary "worst case" of 10 was chosen to include the 3 cases linked to vCJD-infected donors, 4 other cases with known transfusion histories and a further margin for error to allow for any possible under-ascertainment of cases or linkages and stochastic effects).

- The Subgroup also accepted new evidence from human and ovine data that supports an infectivity estimate of the order of one Infectious Dose (ID) per unit of nonleucodepleted red cells (Gregori et al. 2011), where an ID is the amount of pathogen required to cause infection in a susceptible recipient. This contrasts the estimate adopted previously (based on rodent models) that there are many IDs per unit of red cells.

- On prevalence of infection, early findings from a further appendix survey being conducted by the HPA suggested a prevalence at least as high as the existing Hilton et al. estimate, not only for the 1960-85 birth cohort, but also in the 1941-60 cohort (Health Protection Agency 2011).

These points had significant implications for modelling. Conceptually, the confirmation that we should be looking for calibrated outputs rather than relying solely on evidencebased inputs is fundamental. The lower infectivity per donation still produces substantial transmission risks per unit transfused, so the reduction in expected case numbers is not dramatic. Nevertheless, a lower infectivity makes it more plausible that leucodepletion could have had a significant effect in reducing number of new infections from 1999 
onward. This is the subject of ongoing lab-based experimental work. The new evidence from the appendix survey increased the challenge of explaining the comparative absence of blood-borne cases. Nevertheless, the Subgroup advised retention of the default assumption that detectable abnormal prion protein in lymphoid tissue is an indicator of infectivity in blood.

Even after allowing for some possible under-reporting of blood-borne cases, calibration requires us to assume that very few infected recipients (e.g. 10\% of MM homozygotes) would develop clinical vCJD within about 10 years. Whilst accepting this point, the Subgroup advised that future models should not necessarily restrict susceptibility to disease, but also consider scenarios in which other individuals might eventually develop clinical vCJD if they survived long enough. Members requested the inclusion of scenarios in which the distribution of incubation periods amongst individuals of a given codon-129 genotype might be bimodal. Specifically, and because the consequences of infection for MM homozygotes is so critical to the appearance of relatively-early clinical cases, we undertook to explore scenarios in which a minority of infected MMs (perhaps as small as $10 \%$ ) developed symptoms within about 10 years, while all other MMs (and all those of other genotypes) typically had much longer incubation periods. In response to such suggestions, the model has been developed further through continuing discussion with the Subgroup (ACDP, 2012a).To explore scenarios in which almost all recipients might have very long secondary incubation periods, we divided MM homozygotes into two groups, with different (means and distributions of) incubation periods. We also made the model more flexible by relaxing the assumption that transmission from an infected unit, both before and after leucodepletion, was certain. We adapted the model to allow onset of infectivity amongst donors to vary by genotype. These changes allowed us to undertake "what-if" exploration of the Subgroup's advice and suggestions.

An illustrative example is given in Figure 4, which is based on a uniform infection prevalence of 1 in 4,000 for donors born between 1941and 1985. Amongst those infected via red cell transfusion, MM homozygotes are split into those with a "short" mean incubation period of 7 years ( $4 \%$ of entire population) and those with a "long" mean incubation period of 25 years (36\% of population). Non-MM homozygotes ( $60 \%$ of 
population) have a mean incubation period of 30 years. We modelled primary infections occurring circa 1990 and allowed for a mean delay of 2 years, 7 years and 9 years in blood becoming infective for "short" MM, "long" MM and non-MM homozygotes respectively. Mean infectivity per unit of red blood cells is taken to be 0.7 IDs before leucodepletion and 0.1IDs after, giving transmission probabilities of approximately $50 \%$ and $10 \%$ respectively using a Poisson dose-response model (for an explanation of Poisson dose-response models in this context, see, for example, Gregori et al. 2004, Det Norske Veritas Consulting 2004 and Gregori et al. 2011).

This example demonstrates that under certain combinations of feasible parameter values, the model can produce scenarios that approximate the number and timing of clinical cases observed to date. These present amongst the (admittedly hypothetical) "short" incubation period MM homozygotes. By contrast, the estimates for other recipients represent one possible future amongst many. At one extreme, susceptibility to clinical disease might be confined to $10 \%$ of MM homozygotes, so that cases amongst the other groups will never be seen. However, Figure 4 illustrates how a larger number of future cases could still be reconciled with what has been seen so far. Clearly, scenarios with very long incubation periods for most recipients allow many infections to take place while maintaining calibration against case numbers. Although a very high proportion of those infected would die of other causes before developing symptoms, such infections would still be of some concern. Whilst recipients of blood components are now debarred from donating, they may still pose a risk of onward infection via surgery or organ / tissue donation.

Illustrative scenarios such as this can serve as a medium for further discussion of specific assumptions. For example, that shown in Figure 4 has inputs on the effect of leucodepletion that might be regarded as optimistic. This can obviously be altered, but any significant change would require some other inputs to change in compensation if the model outputs are to remain calibrated.

The model can also be used to explore the potential impact of counter measures in any given set of scenarios. For example, we can consider the impact on future clinical case 
numbers if the probability of infection per infected unit could be reduced from 2011 onwards, for example through some form of universal prion removal. The limiting case of a reduction to zero provides an upper bound for the potential impact of any intervention brought in now (in the scenario of Figure 4, for example, stopping all further transmissions would reduce the projected number of secondary clinical cases by about $50 \%$, the rest being caused by transmissions that have already occurred). We can also consider the effect of a shift in the demographic of red cell donors towards those donors born from 1996 onward (and so not significantly exposed to BSE), once they become old enough to donate. This less-exposed group will naturally form a gradually-increasing proportion of the donor base, but this could be deliberately accelerated through some targeted efforts to attract and retain this age group. Modelling can help clarify the circumstances in which this might be worthwhile.

\section{Discussion}

The challenge of forming policy in response to the potential transmission of vCJD is a good example of modelling being both potentially valuable and intrinsically difficult. It represents the only way of gauging the potential scale of a problem with no historical precedent in terms of enduring uncertainties. The long lag between infections and clinical cases means that the effectiveness or otherwise of interventions cannot be established through trials or pilot schemes: to wait for sufficient data to accrue before making decisions could be to miss the opportunity to intervene. Yet modelling is difficult because of the lack of data and understanding surrounding the basic parameters and even some of the biological mechanisms and relationships at play.

In contexts where there is such uncertainty, it can be useful to triangulate between different modelling approaches to provide some reassurance that, with similar input parameters and starting assumptions, we are not merely introducing further uncertainty and confounding factors through our choice of model. Nevertheless, important factors may still be omitted. For example, there might be less overlap between the UK donor population and the sections of the general population with primary vCJD infection than assumed within the models as discussed in this paper. 
The number of unproven assumptions that have to be made inevitably undermine the utility of any single estimate for the number of cases to be expected. Indeed, as modellers we might prefer to wait for an easier problem to come along, where the assumptions can be shown to be valid and where the output generated can be advocated for use with a degree of confidence. However, as Operational Researchers working within or for a Government service with policy colleagues that need to make decisions, there is less scope to choose the problem.

That said, the complexities and uncertainties in this area do mean that modelling should be used and presented with considerable care. Importantly, we have used the model to present features of the evolution of the epidemic and its overall scale under various scenarios, rather than exact numbers. Rather than glossing over uncertainties, its value lies in providing a framework for gauging what is plausible, what is consistent with the data and scientific opinion available, and what the consequences of different sets of beliefs are. To inform debate, it was important that the model could be used to demonstrate the incompatibility between combinations of precautionary assumptions and observed case numbers in a relatively simple way. We needed to explore alternative ways of resolving this contrast, rather than attempting to generate future epidemic curves from a statistical fit. We did not investigate all possible scenarios, but rather provided a framework for colleagues and expert advisors to investigate those uncertainties of most central relevance.

The relatively simple model discussed in this paper has been used to good effect in presenting different elements of the problem both to policy colleagues and to expert advisors, in exploring and feeding back the implications of different assumptions and beliefs, and in illustrating the potential impact of some countermeasures. In this regard, our work has made a beneficial contribution to the decision making process. Given the flexible nature of the model, we anticipate that it will continue to be used to explore the implications of new information regarding parameter estimates and that the feasible range of scenarios consistent with observed clinical cases will be revisited as time progresses. There is continued interest amongst the Subgroup in modelling the impact of alternative assumptions. For example, there has been interest in considering vCJD transmission via 
red cell and Fresh Frozen Plasma transfusion separately and in incorporating the prevalence estimates from a new appendix survey (HPA, 2012; ACDP 2012b).

Additionally, output from the model could potentially inform cost benefit analysis of countermeasures considered in the future.

\section{Acknowledgements}

This is an independent report commissioned and funded by the Policy Research Programme in the Department of Health. The views expressed are not necessarily those of the Department. 


\section{References}

ACDP. Minutes from the $1^{\text {st }}$ meeting of the ACDP Transmissible Spongiform Encephalopathy Risk Assessment Subgroup, July 2011. Available at: http://www.dh.gov.uk/ab/ACDP/TSEguidance/DH_125868 [Accessed November $15,2011]$.

ACDP. Minutes from the $2^{\text {nd }}$ meeting of the ACDP Transmissible Spongiform Encephalopathy Risk Assessment Subgroup (2012a).

ACDP. Advisory Committee on Dangerous Pathogens TSE Risk Assessment Subgroup "Position Statement on occurrence of vCJD and prevalence of infection in the UK population" (2012b). Available at: http://transparency.dh.gov.uk/2012/08/13/tserisk-assessment-july-2012/ [Accessed November 15, 2011].

Bennett, P, A Hare, and J Townshend. "Assessing the Risk of vCJD Transmission via Surgery: Models for Uncertainty and Complexity." Journal of the Operational Research Society 56, no. 2 (November 2004): 202-213.

Bennett, P, and Dobra, SA. "Risk Assessment for Variant CJD in Blood Transfusion: a Perspective from the UK." In Creutzfeldt-Jakob Disease: Managing the Risk of Transmission from Blood, Plasma and Tissues, edited by Turner, M L. AABB Press, 2006.

Bishop, M, P Hart, L Aitchison, HN Baybutt, C Plinston, V Thomson, NL Tuzi, et al. "Predicting Susceptibility and Incubation Time of Human-to-human Transmission of vCJD." The Lancet Neurology 5, no. 5 (May 2006): 393-398.

Bruce, M E, R G Will, J W Ironside, I McConnell, D Drummond, A Suttie, L McCardle, et al. "Transmissions to Mice Indicate That 'New Variant' CJD Is Caused by the BSE Agent." Nature 389, no. 6650 (October 2, 1997): 498-501.

Clarke, Paul, and Azra C Ghani. "Projections of the Future Course of the Primary vCJD Epidemic in the UK: Inclusion of Subclinical Infection and the Possibility of Wider Genetic Susceptibility." Journal of the Royal Society, Interface / the Royal Society 2, no. 2 (March 22, 2005): 19-31.

Collinge, J. "Prion Diseases of Humans and Animals: Their Causes and Molecular Basis." Annual Review of Neuroscience 24, no. 1 (March 2001): 519-550.

Collinge, J, Jerome Whitfield, Edward McKintosh, John Beck, Simon Mead, Dafydd J Thomas, and Michael P Alpers. "Kuru in the 21st Century--an Acquired Human Prion Disease with Very Long Incubation Periods." The Lancet 367, no. 9528 (June 24, 2006): 2068-2074.

Department of Health. Blood-borne Transmision of $v C J D$ : Re-examination of Scenarios, 2011. http://www.dh.gov.uk/health/2011/09/vcjd-scenarios/.

Department of Health. Exclusion of Blood Component Recipients from Donation: The Impact on Potential vCJD Transmission Risks. Publication, July 1, 2006. http://www.dh.gov.uk/en/Publicationsandstatistics/Publications/PublicationsPolic yAndGuidance/DH_4136942.

Department of Health. Mapping Out the Consequences of Screening Blood Donations for PrPSc, 2009.

http://www.dh.gov.uk/en/Publicationsandstatistics/Publications/PublicationsPolic yAndGuidance/DH_094804. 
Det Norske Veritas Consulting, 2004. Risk of infection from variant CJD in blood. 2004. Available at: http://www.dh.gov.uk/en/Publicationsandstatistics/Publications/ PublicationsPolicyAndGuidance/DH_093044 [Accessed July 18, 2011].

Dodd, Roger Y. "Prions and Precautions: Be Careful for What You Ask." Transfusion 50, no. 5 (May 1, 2010): 956-958.

Garske, Tini, and Azra C Ghani. "Uncertainty in the Tail of the Variant Creutzfeldt-Jakob Disease Epidemic in the UK." PloS One 5, no. 12 (2010): e15626.

Ghani, Azra C., Neil M. Ferguson, Christl A. Donnelly, and Roy M. Anderson. "Predicted vCJD Mortality in Great Britain." Nature 406, no. 6796 (2000): 583584.

Ghani, Azra C., Neil M. Ferguson, Christl A. Donnelly, Thomas J. Hagenaars, and Roy M. Anderson. "Epidemiological Determinants of the Pattern and Magnitude of the vCJD Epidemic in Great Britain." Proceedings of the Royal Society of London. Series B: Biological Sciences 265, no. 1413 (December 22, 1998): 2443 -2452.

Gregori, Luisa, Nancy McCombie, Douglas Palmer, Paul Birch, Samuel O SowemimoCoker, Antonio Giulivi, and Robert G Rohwer. "Effectiveness of Leucoreduction for Removal of Infectivity of Transmissible Spongiform Encephalopathies from Blood." Lancet 364, no. 9433 (August 7, 2004): 529-531.

Gregori, Luisa, Hong Yang, and Steven Anderson. "Estimation of Variant Creutzfeldt-Jakob Disease Infectivity Titers in Human Blood.” Transfusion (2011). http://onlinelibrary.wiley.com/doi/10.1111/j.15372995.2011.03199.x/abstract.

Head, M. W, H. M Yull, D. L Ritchie, M. T Bishop, and J. W Ironside. "Pathological Investigation of the First Blood Donor and Recipient Pair Linked by Transfusion-associated Variant Creutzfeldt-Jakob Disease Transmission." Neuropathology and Applied Neurobiology 35, no. 4 (August 1, 2009): 433-436.

Health Protection Agency. Interim Data from the Current National Survey of Abnormal Prion Prevalence in Archived Appendix Specimens, September 2011. http://www.hpa.org.uk/hpr/archives/2011/news3611.htm\#cjd.

Hewitt, P. E., C. A. Llewelyn, J. Mackenzie, and R. G. Will. "Creutzfeldt-Jakob Disease and Blood Transfusion: Results of the UK Transfusion Medicine Epidemiological Review Study." Vox Sanguinis 91, no. 3 (October 2006): 221-230.

Hill, A F, M Desbruslais, S Joiner, K C Sidle, I Gowland, J Collinge, L J Doey, and P Lantos. "The Same Prion Strain Causes vCJD and BSE." Nature 389, no. 6650 (October 2, 1997): 448-450, 526.

Hilton, David A, Azra C Ghani, Lisa Conyers, Philip Edwards, Linda McCardle, Diane Ritchie, Mark Penney, Doha Hegazy, and James W Ironside. "Prevalence of Lymphoreticular Prion Protein Accumulation in UK Tissue Samples." The Journal of Pathology 203, no. 3 (July 2004): 733-739.

Houston, Fiona, Sandra McCutcheon, Wilfred Goldmann, Angela Chong, James Foster, Silvia Sisó, Lorenzo González, Martin Jeffrey, and Nora Hunter. "Prion Diseases Are Efficiently Transmitted by Blood Transfusion in Sheep." Blood 112, no. 12 (December 1, 2008): 4739 -4745.

Huillard d'Aignaux, Jérôme N, Simon N Cousens, Jean Maccario, Dominique Costagliola, Michael P Alpers, Peter G Smith, and Annick Alpérovitch. "The 
Incubation Period of Kuru." Epidemiology (Cambridge, Mass.) 13, no. 4 (July 2002): 402-408.

Ironside, J. W. "Variant Creutzfeldt-Jakob Disease: Prion Protein Genotype Analysis of Positive Appendix Tissue Samples from a Retrospective Prevalence Study." BMJ 332 (May 20, 2006): 1186-1188.

Kaski, Diego, Simon Mead, Harpreet Hyare, Sarah Cooper, Ravi Jampana, James Overell, Richard Knight, John Collinge, and Peter Rudge. "Variant CJD in an Individual Heterozygous for PRNP Codon 129." Lancet 374, no. 9707 (December 19, 2009): 2128.

Knight, Richard. "The Risk of Transmitting Prion Disease by Blood or Plasma Products." Transfusion and Apheresis Science 43, no. 3 (December 2010): 387-391.

Lefrère, Jean-Jacques, and Patricia Hewitt. "From Mad Cows to Sensible Blood Transfusion: The Risk of Prion Transmission by Labile Blood Components in the United Kingdom and in France." Transfusion 49, no. 4 (April 2009): 797-812.

Llewelyn, C A, P E Hewitt, R S G Knight, K Amar, S Cousens, J Mackenzie, and R G Will. "Possible Transmission of Variant Creutzfeldt-Jakob Disease by Blood Transfusion." Lancet 363, no. 9407 (February 7, 2004): 417-421.

Peden, A., L. McCARDLE, M. W Head, S. Love, H. J. T Ward, S. N Cousens, D. M Keeling, C. M Millar, F. G. H Hill, and J. W Ironside. "Variant CJD Infection in the Spleen of a Neurologically Asymptomatic UK Adult Patient with Haemophilia." Haemophilia 16, no. 2 (March 1, 2010): 296-304.

Peden, Alexander H, Mark W Head, L Ritchie Diane, E Bell Jeanne, and W Ironside James. "Preclinical vCJD After Blood Transfusion in a PRNP Codon 129 Heterozygous Patient." The Lancet 364, no. 9433 (August 7, 2004): 527-529.

Stevenson, M D, J E Oakley, S E Chick, and K Chalkidou, 2009. The cost-effectiveness of surgical instrument management policies to reduce the risk of vCJD transmission to humans. Journal of the Operational Research Society 60(4), pp. 506-518.

UK NCJDRSU, and UK Blood Services. "Transfusion Medicine Epidemiology Review", 2011. http://www.cjd.ed.ac.uk/TMER/fate.htm.

Wallis, J. P. "Strategies to Reduce Transfusion Acquired vCJD." Transfusion Medicine 21, no. 1 (February 2011): 1-6.

Wallis, Jonathan P, Angus W Wells, John N Matthews, and Catherine E Chapman. "Long-term Survival After Blood Transfusion: a Population Based Study in the North of England." Transfusion 44, no. 7 (July 1, 2004): 1025-1032.

Watkins, NA, Dobra, SA, Bennett, P, Cairns, J, and Turner, ML. "The Management of Blood Safety in the Presence of Uncertain Risk: A United Kingdom Perspective." Transfusion Medicine Reviews In press (2011).

Wells, A. W, C. A Llewelyn, A. Casbard, A. J Johnson, M. Amin, S. Ballard, J. Buck, M. Malfroy, M. F Murphy, and L. M Williamson. "The EASTR Study: Indications for Transfusion and Estimates of Transfusion Recipient Numbers in Hospitals Supplied by the National Blood Service." Transfusion Medicine 19, no. 6 (December 1, 2009): 315-328.

Will, Bob. "Variant CJD: Where Has It Gone, or Has It?" Practical Neurology 10, no. 5 (October 2010): 250-251. 
Wroe, Stephen J, Suvankar Pal, Durrenajaf Siddique, Harpreet Hyare, Rebecca

Macfarlane, Susan Joiner, Jacqueline M Linehan, et al. "Clinical Presentation and Pre-mortem Diagnosis of Variant Creutzfeldt-Jakob Disease Associated with Blood Transfusion: a Case Report." Lancet 368, no. 9552 (December 9, 2006): 2061-2067. 